\title{
Energy System Modeling showing the benefits of coupling the energy sectors
}

\author{
M. Metzger
}

Published online November 22, 2017

(C) Springer-Verlag GmbH Austria, ein Teil von Springer Nature 2017
The Energiewende towards a share of $80 \%$ renewable energy (REN) related to electricity generation and an overall coverage of $60 \%$ REN with regard to end energy consumption for 2050 leads to new requirements concerning energy infrastructures and markets. The combined consideration of the electricity, thermal and gas sector is a promising option to unlock efficiency potentials for the overall energy supply. However, the analysis of potential multi-modal supply concepts for REN-dominated energy systems requires new modeling approaches and methods. Therefore, the objective of the Energy System Development Plan (ESDP), which is currently conducted by Siemens Corporate Technology and the Institute for High Voltage Technology at RWTH Aachen, is to develop a methodology for an integrated assessment and prediction of energy systems in different regions. This will help to understand the requirements of future energy systems in terms of technologies, information and communication and the associated infrastructure needs.

In a first step, different scenarios for the design of the German energy system, in particular with respect to the variances in generation and utilization, are developed. The assumptions and scenarios are the basis for a subsequent analysis of combined infrastructure and technology options. These are validated by means of a European Multi-Modal Market Simulation. The assessment includes transmission and distribution grid simulations and an economic as well as an ecologic evaluation. The basic transferability of methods and models to other regions and their unique energy systems is a further important objective of the ESDP. In a first phase the ESDP methodology is tested for Germany in the frame of the German "Energiewende" for 2035 and beyond.

Decentralization of power generation is a worldwide trend that is already influencing and will considerably change our energy system and market environment. In addition, there are (electric and thermal) storage and flexibility potentials in the sectors households, small and medium businesses and industry, which can help optimize the energy system in the future. The ESDP modeling approach basically consists of a bottom-up layer for all distributed energy structures and a top-down layer to model central power stations, bulk storage, centralized power to heat units for district and process heating and other centralized generation and conversion facilities as well as cross-border electricity exchanges. The bottomup layer is modeled by using high-resolution geo-data and a comprehensive building database for Germany on the basis of highresolution postal codes. Industrial demands for power and heat are also modeled in a high spatial and temporal resolution and under consideration of different temperature levels for an adequate technology selection. Linking both, the bottom-up and the top-down layer, enables an activation and utilization of decentralized flexibilities.

The presentation will introduce the modeling approach and the underlying assumptions. The technical assessment includes generation aspects as well as an evaluation of transmission and distribution grids.
Kurzfassung eines Vortrags bei der 55. Fachtagung der Österreichischen Gesellschaft fü Energietechnik (OGE) im OVE, die am 12. und 13. Oktober 2017 in Salzburg stattfand.

Metzger, Michael, Siemens AG, Otto-Hahn-Ring 6, 81739, München, Deutschland (E-mail: michael.metzger@siemens.com) 